\title{
Time-communication trade-offs for minimum spanning tree construction
}

\author{
Ali Mashreghi * \\ Department of Computer Science \\ University of Victoria, BC, Canada \\ ali.mashreghi87@gmail.com
}

\author{
Valerie King * \\ Department of Computer Science \\ University of Victoria, BC, Canada \\ val@uvic.ca
}

\begin{abstract}
This paper concerns the problem of constructing a minimum spanning tree (MST) in a synchronous distributed network with $n$ nodes, where each node knows only the identities of itself and its neighbors. We assume the CONGEST model where messages are of size $O(\log n)$ bits. Spanning tree construction was long believed to require an amount of communication linear in the number of edges. In 2015, King, Kutten and Thorup presented a Monte Carlo algorithm which broke this communication bound. In particular it showed that an MST could be constructed with time and message complexity $O\left(n \log ^{2} n / \log \log n\right)$, independent of the number of edges.

Here we give trade-offs between time and communication. Our Monte Carlo algorithm runs in $O(n / \epsilon)$ time and $O\left(\frac{n^{1+\epsilon}}{\epsilon} \log \log n\right)$ messages for any $1>\epsilon \geq \log \log n / \log n$. For the spanning tree problem, we show a time bound of $O(n)$ and a communication bound of $O(n \log n \log \log n)$ messages. We also provide the first algorithm that constructs an MST in time proportional to the diameter of the MST up to a logarithmic factor with $o(m)$ communication.
\end{abstract}

\section{Keywords}

CONGEST; Minimum spanning tree; Randomization; Time complexity; Message complexity

\section{INTRODUCTION}

The problem of broadcasting over a distributed network algorithms efficiently in terms of time and communication is a fundamental problem which has been considered by many researchers over the last 35 years. If we consider the network as an undirected graph with $n$ nodes and $m$ edges, then the spanning tree (or broadcast tree) allows one node to broadcast to $n-1$ other nodes in a network using only $n-1$ messages. If each link is weighted, e.g., by the energy

${ }^{*}$ Funded with an NSERC discovery grant Algorithm Design for Large Graphs and Communications Networks.

Permission to make digital or hard copies of part or all of this work for personal or classroom use is granted without fee provided that copies are not made or distributed for profit or commercial advantage and that copies bear this notice and the full citation on the first page. Copyrights for third-party components of this work must be honored. For all other uses, contact the owner/author(s).

ICDCN '17 January 04-07, 2017, Hyderabad, India

(C) 2017 Copyright held by the owner/author(s).

ACM ISBN 978-1-4503-4839-3/17/01.

DOI: http://dx.doi.org/10.1145/3007748.3007775 needed to send a message over the link, then the minimum spanning tree gives a way to perform the broadcast using the least amount of energy. We show how to efficiently construct a minimum spanning tree (or forest, if the graph is not connected) in a distributed network. Our algorithms are Monte Carlo, and succeed with high probability in the synchronous CONGEST model, where each node may send a message of $O(\log n)$ bits to each of its neighbors in a single round.

A simple algorithm for the spanning tree problem is to construct a breadth-first search tree, by having the root node send to its neighbors and these nodes send to their neighbors, etc., adding an edge to each newly discovered node. This takes optimal time, proportional to the graph diameter, and uses $O(m)$ communication. The classic algorithm by Gallager, Humblet and Spira (GHS) for finding an MST in a distributed (asynchronous) [GHS83] takes $O(n \log n)$ rounds and uses $O(m+n \log n)$ messages. A lower bound of $m$ bits of communication was long conjectured, and has been shown in various models. This bound does not apply to the "standard" model, also called the $K T_{1}$ model in Peleg [Pel00], where each node knows its unique identity and those of its neighbors and the weights of the edges incident to it, if identities can be manipulated arbitrarily. In 2015, King, Kutten and Thorup (KKT) [KKT15] gave Monte Carlo algorithms which rely on performing simple arithmetic operations on identities to go below the bound with both message and time complexity $\tilde{O}(n)$. In particular, their algorithm for minimum spanning tree has time and message complexity $O\left(n \log ^{2} / \log \log n\right)$, independent of the number of edges.

The running time of GHS was improved by Chin and Ting, [CT85], Gafni [Gaf85], and then Awerbuch [Awe87], who found an $O(n)$ time algorithm with $O(m)$ messages. In GHS and these algorithms, nodes are merged to build increasingly large subtrees of the MST, known as fragments. A node determines if an incident edge is outgoing from its current fragment by querying the other endpoint of the edge, where the incident edges are considered sequentially in increasing order of weight. Therefore, finding an outgoing edge from a fragment may take a time proportional to the size of the fragment. This then is a bottleneck for the running time of these techniques, which can be as large as $n$ and much larger than the diameter of the MST.

An alternative approach is for a node to query these edges in parallel but this results in increased communication. A series of works reduced time to $o(n)$ but with $\omega(m)$ messages. These make use of a breadth-first search tree to communicate quickly. Let $D$ is the network's diameter: 
Garay et al. [GKP98] introduced an $O\left(D+n^{0.614}\right)$ time algorithm. Kutten and Peleg [KP95] improved this to time $O\left(D+\sqrt{n} \cdot \log ^{*} n\right)$. In the LOCAL model where messages are of unbounded size, Elkin [Elk04], pointed out another method where each edge is checked to determine if it is the heaviest on any cycle. This takes time proportional to the $M S T$ - radius, which is the maximum over all non-MST tree edges $e$, of the minimum length of a cycle in which $e$ is the heaviest edge; it may be much smaller than $D$. Elkin [Elk04] presented an $\tilde{O}(M S T-$ radius $+\sqrt{n})$ time algorithm for the CONGEST model. Recently, Panduragan et al. [PRS16] gave an algorithm that simultaneously matches the time lower of $\tilde{\Omega}(D+\sqrt{n})$ [Elk06] and the message lower bound of $\Omega(m)\left[\mathrm{KPP}^{+} 15\right]$ in the $K T_{0}$ model.

Our paper explores the question of whether MST construction can be sped up to linear or sublinear time in $n$ and still use only $o(m)$ messages. We show how to construct an MST in $\tilde{O}(\operatorname{diam}(M S T))$ time with $\tilde{O}(n)$ messages, where $\operatorname{diam}(M S T)$ denotes the maximum hop diameter (unweighted diameter) of any MST in the network. When $\operatorname{diam}(M S T)$ is $o(\sqrt{n})$, this is less than the lower bound for time of $\Omega(D+\sqrt{n} / \log n)\left[\mathrm{SHK}^{+} 12\right]$ in the CONGEST model, which holds for Monte Carlo algorithms. However, our result does not contradict this lower bound, as the examples used to establish that bound have MSTs with diameter $\sqrt{n}$. Note that GHS can be modified to run in $\tilde{O}(\operatorname{diam}(M S T))$ time with $O(m)$ messages (see [PRS16]). Our key idea is to use the KKT method to find outgoing edges which avoids the time/communication bottleneck described above.

\subsection{Other related work}

A similar model to CONGEST model is the Congested Clique model. In this model, nodes in the network can directly communicate to each other through a complete underlying graph. Graph connectivity and minimum spanning tree problems have been studied in this model as well. Lotker et al. [LPSPP05] which provided an algorithm to compute the MST in $O(\log \log n)$ rounds. This was improved to an $O(\log \log \log n)$-round algorithm due to Hegemen et al. $\left[\mathrm{HPP}^{+} 15\right]$. Their algorithm is a randomized Monte Carlo which uses Linear Sketches technique to encode the neighborhood of each node, in a manner similar to MST-KKT. Ghaffari and Parter [GP16] have recently improved the time to $O\left(\log ^{*} n\right)$ rounds. The reason for this low number of rounds is that the diameter of the communication network is one and information can be quickly transferred. Therefore, following these ideas directly in the simple CONGEST model will introduce a factor of $O(n)$ to the time complexity. The two mentioned algorithm still use $\Theta\left(n^{2}\right)$ messages. However, in $\left[\mathrm{HPP}^{+} 15\right]$ an algorithm is presented that uses $O(n \cdot \operatorname{polylog}(n))$ messages and $O(\operatorname{polylog}(n))$ rounds. The algorithms for the Congested Clique model do not seem to provide a way to find a linear or near linear time algorithm for MST construction with $o(m)$ messages in the general CONGEST model.

\subsection{Our Results}

We prove the following theorems in the synchronous CONGEST model where each node knows its neighbors and the weights of edges incident to it, and integer weights no greater than $n^{k}$ for $k$ a constant.

THEOREM 1. An MST can be constructed with high prob- ability in a network with $n$ nodes in

1. $O\left(\operatorname{diam}(M S T) \frac{\log ^{2} n}{\log \log n}\right)$ time and $O\left(n \frac{\log ^{2} n}{\log \log n} \log \operatorname{diam}(M S T)\right)$ messages;

2. $O(n / \epsilon)$ time and $O\left((1 / \epsilon) n^{1+\epsilon} \log \log n\right)$ messages where $\log \log n / \lg n \leq \epsilon<1$.

According to the second part of this theorem, choosing a constant value for $\epsilon$, e.g. 0.1 , we can a have linear time and $O(n)$ and $O\left(n^{1.1} \log \log n\right)$ messages which could be significantly less than the number of edges.

TheOREM 2. A spanning tree can be constructed with high probability in a network with $n$ nodes in $O(n)$ time and $O(n \log n \log \log n)$ messages.

\subsection{Overview of the algorithms}

First we define some important terms. At any time step of the algorithms a fragment is a subtree of the final MST. Each fragment has a leader which could be any node on that fragment, e.g. the node with smallest ID. Height of a fragment is the maximum number of edges between a node and the leader on that fragment.

Also we define $\operatorname{diam}(M S T)$ to be the hop diameter of the MST which is the maximum number of edges on any path in the MST. Hop diameter of a minimum spanning forest is the maximum hop diameter over all of its spanning trees. Let KKT denote the MST algorithm in [KKT15], the MST is built up in iterations, from a set of fragments which are disjoint subtrees of the MST. Initially, each node is a fragment. In each iteration, fragments find outgoing edges with minimum weight and merge through these outgoing edges until the MST is found. We observe that the time to find a minimum weight outgoing edge in one fragment is proportional to the height of the fragment (times $O\left(\frac{\log n}{\log \log n}\right)$ ). Height of a fragment is defined to be. The KKT runs for $O(\log n)$ iterations; each iteration is assumed to require the worst case time, i.e., that the height of some fragment in the iteration is $n$.

To achieve $\tilde{O}(\operatorname{diam}(M S T))$ time for constructing an MST, it suffices to alter KKT so that it is run in phases, with increasing larger guesses as to the diameter of the MST. Initially, the diameter of the MST is assumed to be a constant. Each fragment verifies with high probability that it has no outgoing edges. If it does, the diameter is doubled and the algorithm is repeated.

To achieve $O(n / \epsilon)$ time for finding the MST is a bit more complicated. We modify KKT so that an iteration on a fragment of height $T$ takes time proportional to its height. We apply a threshold $T$ so that only fragments that have height less than $T$ are permitted to look for outgoing edges. Once enough iterations are executed so that all fragments with height less than $T$ are merged, we are left with a small number of fragments. This allows us to apply fewer iterations to merge them. We repeat until the final MST remains. The algorithm runs in less than $\log ^{*} n$ phases. For the first phase $i=1$, we apply a threshold of $T=n /(\log n)^{2}$ and run for $\log n$ iterations. For the following phases $i=2,3, \ldots, \log ^{*} n$, we apply a threshold $T(i)=\frac{n}{\left(\log ^{(i)}\right)^{2}}$ and run $O\left(\log ^{(i)}\right)$ iterations, where $\log ^{(i)} n$ denotes $i$ iterated logarithms. Each iteration requires $n / \epsilon$ time and the total time is therefore $O\left((n / \epsilon) \sum_{i} 1 / \log ^{(i)}\right)$.

The thresholding idea is implemented in a manner similar to [Awe87]. There are two other key points here: 
1. The time complexity of the KKT is $O\left(n \log n \frac{\log n}{\log \log n}\right)$. The application of thresholds removes one $\log n$ factor. The factor $O\left(\frac{\log n}{\log \log n}\right)$ is due to a $\log n$-ary search for finding the lightest outgoing edge. In order to reach the overall time complexity, we modify FindMIN-C from KKT to run an $n^{\epsilon}-$ ary search using a pipelined broadcast-and-echo which increases communication by an $O\left(n^{\epsilon} / \log n\right)$ factor and replaces the $O\left(\frac{\log n}{\log \log n}\right)$ time factor by $O(1)$ for constant $\epsilon$.

2 . In each iteration of KKT, there is a constant probability of reducing the number of fragments by a constant factor. An expected $O(\log n)$ iterations are needed to construct the MST. With an additional $O(\log n)$ iterations, KKT succeeds with high probability. In order to get high probability of merging all trees with heights below $T$ for each phase, we use $O(\log n)$ parallel repetitions of each iteration instead of just one. This does not affect the search time; however, it increases communication by a factor of $\log n$.

We construct a spanning tree in linear time but with less communication than we use for MST construction. As in the MST construction, we use gradually increasing thresholds on the amount of time for each iteration, but in the spanning tree, outgoing edges may form a cycle of fragments. The problem of breaking the cycle in time proportion to the threshold requires a somewhat technical solution.

\subsection{Organization of the paper}

Section 2 describes the preliminaries and reviews the algorithms of [KKT15]. Section 3 shows the modified version of KKT which works in $\tilde{O}(\operatorname{diam}(M S T))$ time rounds. Section 4 provides the linear time algorithm minimum spanning tree construction. In Section 5, the spanning tree algorithm is described. In Section 6, the conclusion and open problems are presented.

\section{PRELIMINARIES}

\subsection{The model}

The network is an undirected graph $G=(V, E)$ in which $|V|=n,|E|=m$, and messages of $O(\log n)$ bits are allowed. We assume the CONGEST model: Communication proceeds in synchronous rounds (also known as time steps); a message that is sent through a link at the beginning of the round will be received by the end of the round; and in one round a node may send a message to each of its neighbors. Initially each node knows only is its own ID plus the the ID's of its neighbours and the weights of the edges to its neighbors $\left(K T_{1}\right.$ model in [Pel00]).

All nodes know the same upper bound on the network size, i.e., $n$, and all nodes start at time 0 . Edge weights are positive integers less than $n^{k}$ where $k$ is any constant. Note that edge weights can be made unique simply by concatenating the ID's of the nodes to end of edge weights; hence for the remainder of the paper, we assume they are unique. As a result, our network has a unique MST.

\subsection{Review of $\mathrm{KKT}$ and $\mathrm{ST}-\mathrm{KKT}$}

Throughout the paper KKT and ST-KKT refer to the algorithms in [KKT15] for minimum spanning tree and spanning tree, respectively. These algorithms rely on a basic observation: Every edge with both endpoints in a fragment contributes 2 to the sum of degrees of nodes in the fragment, while an outgoing edge contributes 1 . Hence if the parity of this sum is odd, there must be an outgoing edge.
Here we describe some subroutines from KKT [KKT15] that we will use directly or modify.

Broadcast-and-echo(F): A basic communication pattern in which a leader of a fragment $F$ broadcasts a message to all nodes in the fragment (broadcast) and in response all the nodes (starting from leaves) send up a message towards the leader (echo).

Odd hash function A function $h:[1, m] \rightarrow\{0,1\}$ such that for any non-empty set $S \subseteq[1, m]$, with probability $1 / 8$, hashes an odd number of members from $S$ to 1. In particular, let us say that for some fragment, $S$ is the multiset of all edges connected to the nodes of that fragment. If this fragment has at least one outgoing edge then with probability $1 / 8, \sum_{e \in S} h(e)$ $\bmod 2=1$. This is because any edge whose both endpoints are in the fragment will appear twice and will not contribute to the sum. Therefore, by computing this sum in a fragment with constant probability we can say whether it has an outgoing edge.

TestOut $(x, I)$ : With constant probability, returns true if there is an outgoing edge leaving $F_{x}$ (the fragment of leader $x$ ) whose weight is in interval $I$, and false otherwise. TESTOUT uses an odd hash function to test if there is an outgoing edge in the given interval.

HP-TestOut $(x, I)$ : Does the same thing as TestOut except with high probability.

FindMin- $\mathbf{C}(x)$ : Finds the lightest outgoing edge from a fragment $F_{x}$ with constant probability, using worst case $O\left(\left|F_{x}\right| \log n / \log \log n\right)$ messages and time. If there is no edge leaving the tree FINDMIN-C always returns $\emptyset$. It works by partitioning the whole search range $\left(n^{k}\right)$ each time into $\log n$ equal sized intervals and obtaining the result of TESTOUT for each interval. Then the first interval that has an outgoing edge is picked and partitioned again. Repeating this, after $\log _{\log n} n^{k}=O\left(\frac{\log n}{\log \log n}\right)$ iterations the lightest outgoing edge is found.

FindAny-C $(x)$ : Finds any outgoing edge from a fragment $F_{x}$ with constant probability, using worst case $O\left(\left|F_{x}\right|\right)$ messages and time (one broadcast-and-return). If there is no edge leaving the tree, it always returns $\emptyset$. We note that the broadcast requires a $O(\log n)$ bit transmission of a hash function and the return requires the return of an $O(\log n)$ bit word.

KKT works in $O(\log n)$ phases. In each phase, every fragment leader uses FindMin-C to find the lightest outgoing edge with constant probability. Afterwards, the fragments merge using the lightest outgoing edges they have found. This is shown to divide the number of fragments by a constant each time; hence, having $O(\log n)$ phases overall. FindMin-C works by broadcasting an odd hash function and an interval which is to be divided to $O(\log n)$ equal sized subintervals. For each subinterval in parallel, TestOut is applied to find the first subinterval that has an outgoing edge. Therefore, the dividing process is repeated for this subinterval until the minimum outgoing edge is located. HP-TESTOUT is used to verify the results returned by TestOut. 


\section{MST WITH SMALL DIAMETER}

In this section we present a modification of KKT which takes $O\left(\operatorname{diam}(M S T) \frac{\log ^{2} n}{\log \log n}\right)$ time and uses sublinear communication in the number of edges when $m$ is sufficiently large.

Each iteration of KKT allows the fragments $O(n)$ time rounds to look for outgoing edges. However, we know that over the course of algorithm each fragment is actually a part of the final MST. This implies that if the diameter of the MST is low then the diameter of any fragment is low as well. In $O(\operatorname{diam}(M S T))$ time fragments can find lightest outgoing edges. However, we do not know MST's diameter beforehand; hence, we have to guess. In particular, we start from a constant estimate of the diameter of the MST, and simulate a version of KKT assuming our estimation is an upper bound on diameter. We find the MST as soon as our estimation $\hat{D}$ for $\operatorname{diam}(M S T)$ is greater than $\operatorname{diam}(M S T)$. When we estimate $\hat{D}$, we prevent fragments from spending time more than $O(\hat{D})$.

We use the thresholding technique to distinguish between fragments with height larger than the desired threshold $T$ on the height of fragments and those with height less than $T$. Let active fragments be those allowed to look for an outgoing edge, i.e., those with height less than $T$. For the purpose of distinguishing, before starting an iteration of KKT we do as follows.

The algorithm runs in iterations which are timed according to the global clock. At the start of each iteration all leaders deactivate themselves. Every leaf makes a timer message and sends it upward. A timer is a message with the initial value of threshold $T$ and each time it passes a link its value is decreased by one, and once the value hits zero it will stop being transmitted. Timer message is very similar to the exploration token used by Awerbuch in [Awe87]; however, here, a timer does not try to measure size of a fragment and only cares about the height of a fragment which is the deciding factor for time complexity.

When all the leaves (in all fragments) sent up their timers, every internal node waits for the timers from all its children and when it has received all of them, it sends up the timer with minimum value. Consequently, the leader of each fragment will receive the timers of all of its children if and only if the height of the fragment is no greater than the threshold. If the height of the fragment is no more than $T$ the leader will be activated (along with its fragment) and it can start to look for outgoing edges in the upcoming KKT iteration. Algorithm 1 shows a pseudo-code of this activation process.

Now using the ACTIVATE procedure we can present the algorithm DiAM-KKT which only takes $O\left(\operatorname{diam}(M S T) \frac{\log ^{2} n}{\log \log n}\right)$ time. The algorithm starts by setting the estimation of $\operatorname{diam}(M S T)$, i.e. $\hat{D}$, to 1 . Afterwards, before running an iteration of KKT it first activates only fragments whose height is less than $\hat{D}$. Intuitively, we are assuming that $\hat{D}$ is the real value of $\operatorname{diam}(M S T)$; hence, there is no point in allowing fragments with height bigger than $\hat{D}$ to look for outgoing edges. Knowing this, if we were proved wrong and could not find the MST we will make a higher estimation by doubling $\hat{D}$. After performing enough iterations, i.e. $O(\log n)$, we need to see if we could find the minimum spanning tree/forest or not. To do this, we actually do not need to know the exact size of network; we can test w.h.p the existence of outgoing edges using a verification method. As soon as our estimate is good enough, i.e. $\operatorname{diam}(M S T) \leq \hat{D}<2 \cdot \operatorname{diam}(M S T)$, w.h.p the MST is found. If the minimum spanning tree or forest is found, in step 9 of DiAm-KKT, no fragment can have outgoing edges; therefore, all nodes of the network receive the message STOP and the algorithm successfully terminates. Next theorem will bound time and message complexity of DIAMKKT.

Theorem 3. DiAm-KKT uses $O\left(\operatorname{diam}(M S T) \frac{\log ^{2} n}{\log \log n}\right)$ time and $O\left(n \frac{\log ^{2} n}{\log \log n} \cdot \log \operatorname{diam}(M S T)\right)$ messages w.h.p.

Proof. W.h.p the algorithm finishes as soon as $\hat{D} \geq$ $\operatorname{diam}(M S T)$. Thus, w.h.p. we have a total of $\log \operatorname{diam}(M S T)$ estimations since each time we are doubling $\hat{D}$, and factor $\log \operatorname{diam}(M S T)$ appears in message complexity. However, in the time complexity, apart from the log factors, each time we are doubling the time allowed for communication. Therefore, sum of this geometric sequence is dependent on the last term which is, w.h.p. at most twice the real value of the MST's diamter, i.e. $2 \cdot \operatorname{diam}(M S T)$. Besides, we have $O(\log n)$ iterations, and a multiplicative factor of $O\left(\frac{\log n}{\log \log n}\right)$ due to the $\log n$-ary search of FindMin-C. Hence, the overall time complexity is $O\left(\operatorname{diam}(M S T) \frac{\log ^{2} n}{\log \log n}\right)$.

\section{LINEAR TIME ALGORITHM FOR CON- STRUCTING THE MST}

DIAMKKT is a very fast algorithm if the diameter of the MST is small enough. However, if $\operatorname{diam}(M S T)>\frac{n \log \log n}{\log ^{2} n}$ then it will take $\omega(n)$ time rounds. Here, we want to present an algorithm for MST construction which always works in linear time and takes sublinear communication in the number of edges. We again use $T$, a threshold bounding the height of the fragments looking for outgoing edges.

To this end, we must find a way to detect the minimum outgoing edge in $O(T / \epsilon)$ rounds. In fact, we want to get rid of the $\log _{\log n} n$ (or $\frac{\log n}{\log \log n}$ ) that appears in FindMin-C due to a $\log n$-ary search. As before, we assume that the maximum edge weight is less than $n^{k}$ for some constant $k$. In KKT the range of edge weights is partitioned into $\log n$ equal sized intervals; hence, $\frac{\log n}{\log \log n}$ narrowing downs are needed to find the minimum outgoing edge. To speed up the process, we narrow down the interval by a factor of $n^{\epsilon}$ each time, where $n^{\epsilon}>\log n$.

Similar to KKT, for the $i^{\text {th }}$ interval we need one bit to be the result of TestOut $\left(x, I_{i}\right)$, where $I_{i}$ is the $i^{\text {th }}$ part of the interval that we are searching. Then, the leader will pick the first part that has an outgoing edge, i.e. the first interval whose TESTOUT result is true, and again this new interval will be divided by $n^{\epsilon}$.

Nevertheless, in order to do this, each node needs an array of $n^{\epsilon}$ bits. Sending an array with this magnitude will need $\frac{n^{\epsilon}}{\log n}$ consecutive messages. Therefore, we use a pipelining technique so that every leaf sends these messages one after another without delay, and every internal node upon receiving the array from all its children calculates the sum of those arrays and sends it up. In fact, internal nodes will receive the parts of their children's arrays in order; therefore, they can compute the sum for the parts they have received (from 

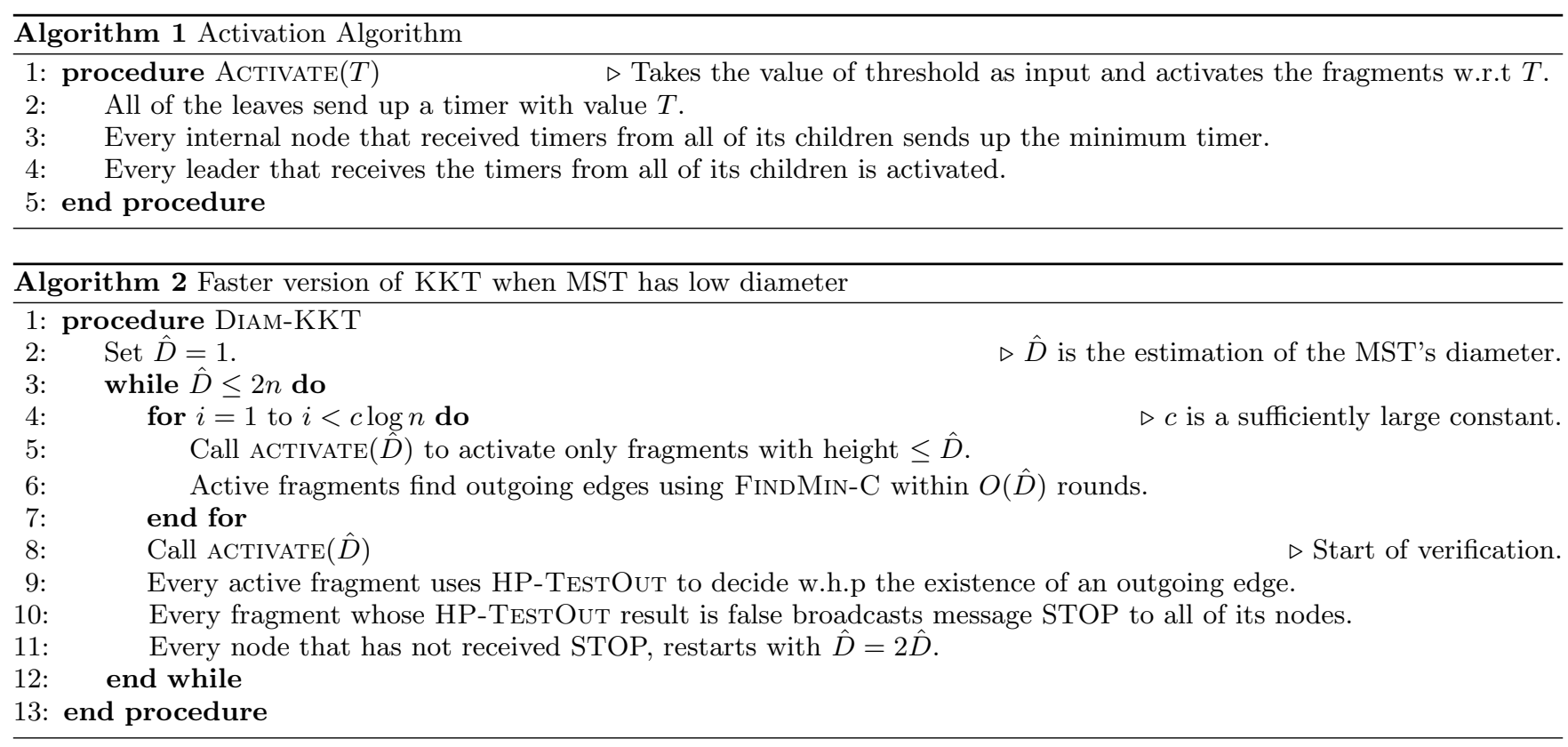

all children) and send it up without waiting for the whole array to arrive.

It is easy to verify that the time rounds needed for the leader to receive the whole array from all its children is at most $T+\frac{n^{\epsilon}}{\log n} \leq 2 T$ if $\epsilon<1$, because the height of the fragment cannot be more than $T$, otherwise it would not be allowed to look for outgoing edges.

Now we show how this pipelining technique helps in getting high probability in a single iteration. The idea is that instead of using only one hash function we use $O(\log n)$ pipelined hash functions. But again, we do not wait for the results of the first hash function and then repeat the process with another one. We simultaneously (almost) apply $\log n$ randomly chosen hash functions. The leader broadcasts all of the $O(\log n)$ hash functions one after another and without any delay. This $\log n$ extra messages through each link will not affect the time complexity because the extra $O(\log n)$ time will be added to the threshold on fragment's height, which is relatively very large; this pipelining of hash functions only affects message complexity by a factor of $O(\log n)$. Algorithm FASTFINDMIN shows how finding the lightest outgoing edge can be done in $O(T / \epsilon)$ with high probability using $O(\log n)$ hash functions.

We also modify TESTOuT to take another argument $h_{j}$ which is the hash function that it will use. Note that when we are in the first phase of the algorithm we can have $O(\log n)$ iterations. Therefore, we will get high probability with applying only one hash function in FASTFINDMIN-C. We do not give the pseudocode separately for FASTFInDMin-C. It is FASTFINDMIN but with one hash function instead of $O(\log n)$, and it gets constant probability of success.

LEMMA 1. There exists a constant $c$ for which, FASTFINDMiN finds the lightest outgoing edge w.h.p.

Proof. Imagine $I$ is the current interval under search. Let $I_{f}$ be the first subinterval that has an outgoing edge. Since, as stated in [KKT15], each $h_{i}$ is an odd hash function, the probability that an odd number of outgoing edges in $I_{f}$ hash into 1 is at least $1 / 8$. Note that non-outgoing edges will cancel each other's parity since they appear exactly twice in the sum. Therefore, the probability of this event not happening is less than $7 / 8$ for one hash function. Using $c \log n$ hash functions this probability is reduced to $\frac{7}{8}^{c \log n}$. However, we need to narrow down the interval a total of $k / \epsilon$ times, and the whole process fails if any of these narrowing downs fails. Hence, by union bound, the probability of not finding the lightest outgoing edge will be $\frac{k}{\epsilon} \cdot\left(\frac{7}{8}\right)^{c \log n}$. Now, note that we always want $n^{\epsilon}=\Omega(\log n)$, because otherwise the whole array can be transmitted in one message; this implies that $\epsilon>\Omega\left(\frac{\log \log n}{\log n}\right)$. Thus, for $\frac{k}{\epsilon} \cdot\left(\frac{7}{8}\right)^{c \log n}$ to be less than $1 / n^{c_{1}}$ it suffices that $\left(\frac{7}{8}\right)^{c \log n}<\frac{1}{n^{c_{1}+3}}$ which happens for $c>\frac{c_{1}+3}{\log \frac{8}{7}}$.

Having all the prerequisites, we can provide the FASTMST algorithm which finds an MST in $O(n / \epsilon)$ rounds and with $O\left(\frac{n^{1+\epsilon} \log \log n}{\epsilon}\right)$ communication w.h.p. FASTMST starts with setting the threshold value to $\frac{n}{\log ^{2} n}$. Before executing an iteration for finding outgoing edges the procedure ACTIVATE is called to make sure that only fragments whose height is below threshold are communicating and looking for outgoing edges. For every value of threshold (say $T=\frac{n}{(\log (i) n)^{2}}$ ), $O\left(\log ^{(i)} n\right)$ iterations are performed. Next theorem shows that this many iterations are enough to merge all fragments with height below $T$.

ThEOREM 4. Algorithm FASTMST terminates in $O(n / \epsilon)$ rounds w.h.p.

Proof. We know from Lemma 1 that FASTFindMin fails probability $1 / n^{c_{1}}$ for some constant $c_{1}$. Now, in any iteration, there are at most $n$ fragments. Therefore, by union bound, w.h.p the number of fragments is divided by at least 2 in each iteration. Hence, the number of iterations needed for each phase is log of the number of fragments. Furthermore, there are a total of $\sum_{i=1}^{i=\log ^{*} n} O\left(\log ^{(i)} n\right)=O(\log n)$ iterations and they all succeed w.h.p, again by union bound. The maximum edge weight is $n^{k}$ for some constant $k$ and 

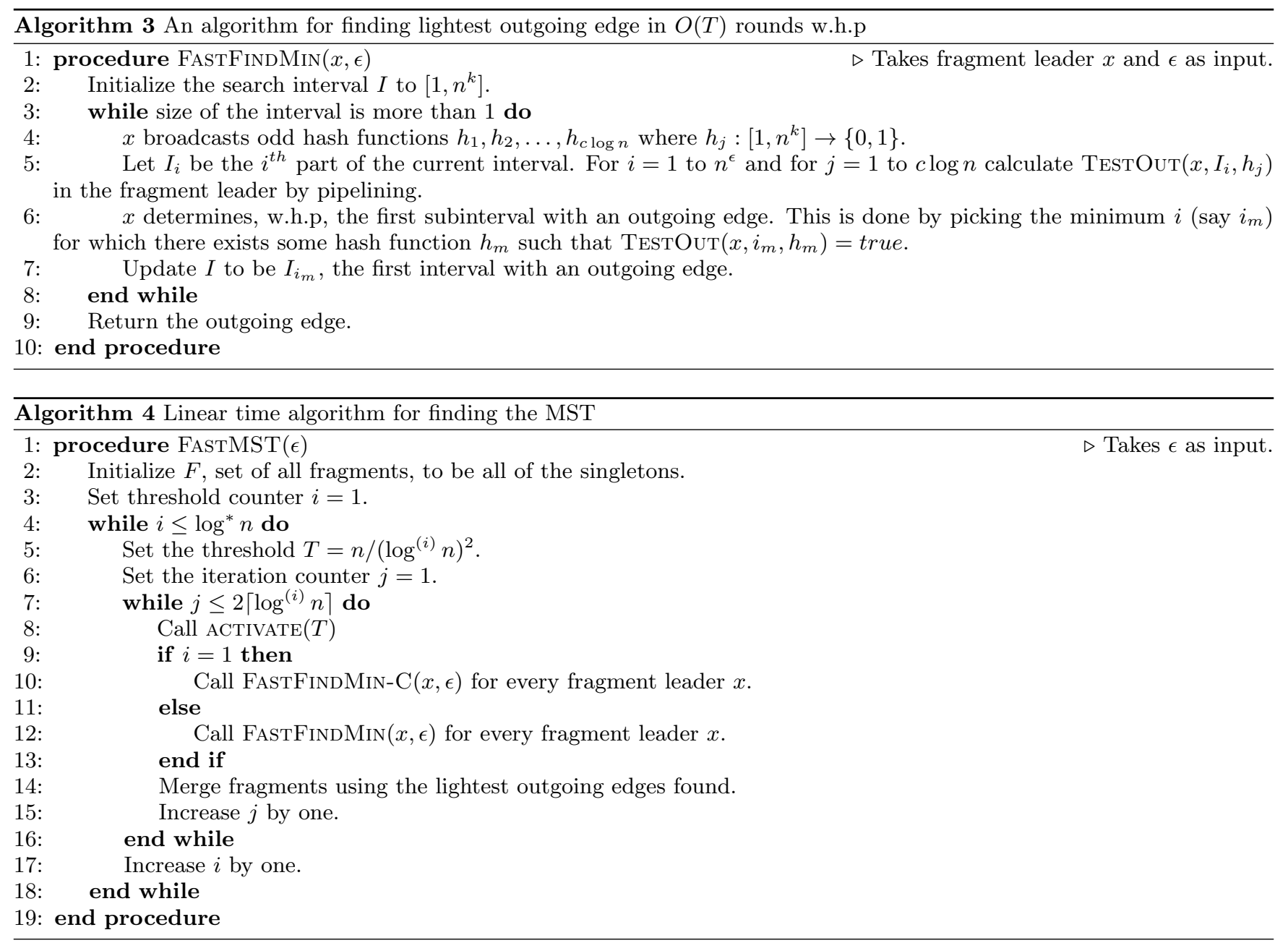

since each time we are narrowing down the range under search by $n^{\epsilon}$ then a total of $\frac{k}{\epsilon}$ narrowing downs are needed; hence the factor $\frac{1}{\epsilon}$ in the time complexity.

When all of the fragments with height $<n /\left(\log ^{(i)} n\right)^{2}$ are merged, the number of remaining fragments cannot exceed $\left(\log ^{(i)} n\right)^{2}$. Therefore, for each phase where the threshold is updated from $n /\left(\log ^{(i-1)} n\right)^{2}$ to $n /\left(\log ^{(i)} n\right)^{2}$, we only need $O\left(\log \left(\log ^{(i-1)} n\right)^{2}\right)=O\left(\log ^{(i)} n\right)$ iterations. Moreover, in the last phase the threshold is exactly $n$ and all of the remaining fragments will merge.

In each iteration only fragments with height below the threshold are allowed to look for outgoing edges. Thus, the overall complexity will be

$\frac{1}{\epsilon} \sum_{i=1}^{i=\log ^{*} n} O\left(\log ^{(i)} n\right) \cdot O\left(\frac{n}{\left(\log ^{(i)} n\right)^{2}}\right)=\frac{n}{\epsilon} \sum_{i=1}^{i=\log ^{*} n} O\left(\frac{1}{\log ^{(i)} n}\right)$.

The last term of this sum is 1 and each time the denominator is losing a log; we can say the denominator is at least doubled each time. Therefore, this sum is less than $\sum_{i=0}^{\infty} \frac{1}{2^{i}}=1$, and the theorem follows.

TheOREM 5. Algorithm FASTMST requires $O\left(\frac{n^{1+\epsilon} \log \log n}{\epsilon}\right)$ messages.

Proof. As stated in Theorem 4, it takes $\frac{k}{\epsilon}$ narrowing downs before the lightest outgoing edge is found. For each of these narrowing downs every node needs to communicate an $n^{\epsilon}$-bit array upward for each of the $O(\log n)$ hash functions. This is $O\left(n^{\epsilon} \log n\right)$ bits which can be pipelined in time $O\left(n^{\epsilon}\right)$. In total, every link will be used for $\frac{k}{\epsilon} \cdot n^{\epsilon}$ messages after the first phase. Besides, there will be $O(\log \log n)$ iterations over all phases $i \geq 2$. On the other hand, in the first phase, we use only one hash function but we have $O(\log n)$ iterations. Thus, considering $k$ is constant, the total message complexity will be:

$O\left(\frac{1}{\epsilon} \frac{n^{1+\epsilon}}{\log n} \log n\right)+O\left(\frac{1}{\epsilon} \frac{n^{1+\epsilon}}{\log n} \log n \log \log n\right)=O\left(\frac{n^{1+\epsilon}}{\epsilon} \log \log n\right)$.

\section{LINEAR TIME ALGORITHM FOR SPAN- NING TREE}

For the construction of spanning tree in linear time we again use a threshold $T$ to only allow fragments with height less than $T$ to look for outgoing edges. Like the previous section we need to boost the probability when we cannot have $O(\log n)$ iterations. This will be done by running repetitions of each iteration in parallel.

However, the difficulty will come when we try to handle cycles. In ST-KKT a node realizes that it is on a cycle if it does not hear from at least one of its children after 
enough time. More precisely, every leaf sends a message upward and every internal node waits to hear from all its children, and if it did not hear from them after enough time (say $O(n)$ rounds) then it must have been on a cycle. In our algorithm, since we are using some threshold we cannot provide enough time to make sure that internal nodes hear from their children. In fact, if an internal node does not hear from all of its children it could be that it is on a cycle or the threshold was not large enough. We need to somehow distinguish between these two cases. In this section we will provide our algorithm for detecting and removing cycles. Throughout this section we assume that each edge has a unique ID made by concatenating the IDs of its endpoints, where the ID of the endpoint with smaller ID comes first.

\subsection{Handling Cycles}

After each fragment finds an outgoing edge, we need to break the cycles, i.e., remove an edge from the cycle. Let us define some important terms first. In iteration $i+1$ of the algorithm we have:

Old fragment: A fragment at the end of the $i^{\text {th }}$ iteration.

Marked edges of a node: When an old fragment $F$ finds an outgoing edge, the endpoint of the outgoing edge in $F$ is called the tail, while the other endpoint is called the head. A node $s$ can be the head of a number of outgoing edges found either on the this iteration or on previous iterations. These edges are considered the marked edges for node $s$ unless they have been removed from the list of marked edges at some point during the algorithm. Note that an outgoing edge is not considered marked for its tail.

Current leader (of an old fragment): In iteration $i+1$ every old fragment tries to find an outgoing edge. If it succeeds in doing so, its current leader will become the tail of the outgoing edge. Otherwise, its current leader is the same as its leader at the end of iteration $i$.

Cluster: When finding outgoing edges is over, consider the graph formed by the old fragments and edges that are marked for some node at the moment. Each cluster is in fact a connected component in this graph. A cluster contains one or more of the old fragments at this iteration.

Bad edge: Is a marked edge which is either on a cycle (of any length) or on a path of length $>T$ from a leaf in its cluster.

1-bad and 2-bad fragments: If an old fragment has 2 or more bad edges in total, it is called a 2-bad fragment. Note that the number of bad edges of an old fragment is the sum of bad edges of its nodes (including the current leader). A 1-bad fragment has exactly 1 bad edge.

Our goal is to keep the invariant that clusters with diameter less than $T$ are merged into fragments with diameter greater than $T$ before $T$ is incremented.

The basic idea of removing cycles in time proportional to the threshold is to remove a cycle only when necessary. Later we will prove that a 2-bad fragment has to belong to a cluster of diameter more than $T$. In fact, we will prove that the current leader of a 2-bad fragment has to be on a path of length more than $T$ from a leaf in the cluster. Therefore, a cycle that lies in cluster containing 2-bad fragments can wait to be removed until the threshold gets big enough. However, we make sure that the cycle of a cluster with only 1-bad fragments is broken.

One problem is that we do not know if the bad edge of a 1-bad fragment is because of a cycle or a long path. Therefore, when we are eliminating edges to get rid of cycles we might be eliminating edges from a long path as well. This is problematic if the eliminated edges are in a cluster which has 2-bad fragments. In this case a 2-bad fragment might become a 1-bad fragment because we have removed an edge from a long path that was connected to it. We have to prevent this, otherwise new cycles of 1-bad fragments keep appearing that we have to deal with. In order to prevent a 2-bad fragment from becoming 1-bad, we eliminate an edge only if it is at distance more than $T$ from the current leader of 2-bad fragments. In particular, 2-fragments signal 1-bad fragments that are within distance $T$ and forbid them from eliminating edges.

In other words, we make sure that cycles in cluster that only have 1-bad fragments are broken, while we guarantee that eliminated edges do not result in more 1-bad fragments. The steps we follow to remove cycles are as follows:

1) Determining bad edges.

2) Current leader determines $B$ the number of bad edges in its old fragment.

3) a) If $B>1$, observe this occurs when there is a path of length more than $T$ from a leaf to the current leader in the cluster. An edge is removed if it is at distance more than $T$ from the current leader of a 2-bad fragment. 2-bad fragments send a ChoiceForbidden signal to 1-bad fragments within distance $T$ and prevent them from eliminating edges. b) If $B=1$, and the current leader has not received any ChoiceForbidden signal, it will choose to eliminate either the outgoing edge or the bad edge, depending on which has the smaller ID. Elimination occurs if an edge is chosen by both its endpoints for removal.

When cycles are broken, all fragments merge using the remaining outgoing edges. Note that there are still clusters which have cycles but these clusters have 2-bad fragments. Consequently, they will not look for outgoing edges until the threshold becomes large enough; hence, they cannot form new cycles. As we will prove later, a cluster cannot have more than one cycle. Therefore, the only cycles of such clusters will be removed with the same technique at higher thresholds.

Before presenting the algorithm for removing cycles we prove that clusters that have 2-bad fragments have to have diameter larger than $T$; hence, they can be dealt with in higher thresholds. The following lemma proves this.

LEMMA 2. If an old fragment $F$ is 2-bad the diameter of its cluster is more than $T$.

Proof. First we observe that if $F$ is on a cycle it has to have an outgoing edge. The reason is that every fragment can have at most one outgoing edge; hence, the fragments connected to $F$ will form a tree like structure unless $F$ itself has an outgoing edge to one the fragments connected (di- 
rectly or indirectly) to it. Now, for the same reason all of these connected fragments cannot have more than one cycle because if they did one of the fragments should have had more than one outgoing edge which is a contradiction. Thus, if we imagine that $F$ has two or more bad incoming edges at most one of them could be due to a cycle and the other must be the result of a long height (compared to threshold) that has prevented some timer to reach high enough. This ensures the existence of path with length more than $T$ in $F$ 's cluster and the lemma follows.

Algorithm CycleRemover shows how to break cycles. When fragments find their outgoing edges, we make sure that cycles in clusters with only 1-bad fragments are removed. We do this carefully so that no 2-bad fragment becomes 1-bad after some edges are eliminated. We assume every node keeps a list of marked edges. All nodes only add to their list of marked edges over the course of algorithm unless they receive a Clear message from the leader which tells them to empty their lists. A node will receive the Clear message when it is no longer on a cycle and the height of its cluster is no more than $T$.

In CyCleRemover, we use timers as in the MST algorithm. Every leaf sends up a timer with value $T$ upward to the current leader. Every internal node (including the current leader) that receives the timer from all of its children sends up the timer with minimum value. As a result, after $T$ rounds every node will know which of its marked edges it has not heard from, i.e. bad edges. Then every node of an old fragment sends up the number of its bad edges to the current leader. Again after $T$ rounds, the current leader will know how many bad edges its fragment has; hence, 1bad and 2-bad fragments are determined. Now, before we break the cycles, we make sure that edges that are within distance $T$ of a 2-bad fragment are not eliminated. To this end, the current leader of every 2-bad fragment sends down the ChoiceForbidden message which is attached to a timer with value $T$. The leader of any 1-bad fragment that receives such a message does not eliminate any edges. In order to prevent redundancy, if a ChoiceForbidden messages reaches the leader of some 2-bad fragment, it will not be forwarded because that leader has already sent down one such message.

1-bad fragments that have not received the ChoiceForbidden message eliminate either the outgoing edge or the bad edge. In particular, the fragment picks edge that has the smaller ID for removal. This edge is removed only if the fragment on the other side picks it for removal, as well. The next lemma shows that eliminating the node with smaller ID will break the cycles, and this does not affect the asymptotic number of iterations we need to merge all the fragments with height below the threshold.

LEMMA 3. If all of 1-bad fragments that have not received the ChoiceForbidden message pick between their bad edge and outgoing edge the one with smaller ID and edges picked by both endpoints are removed, cycles in cluster with only 1-bad fragments will break. This does not affect the asymptotic number of iterations required for merging fragments in the current phase.

Proof. Consider a cycle $C$ that is not broken after calling CyCleRemover. If there is at least one 2-bad fragment in that cycle, that fragment will broadcast the ChoiceForbidden message and prevents the height of the cluster from becoming less than $T$. This whole cluster along with the cycle will be dealt with in higher thresholds. Now, if there is no 2-bad fragment in the cycle, having a cycle itself implies that all of the fragments have to be 1-bad. These 1-bad fragments will not receive any ChoiceForbidden message and hence choose between their outgoing edge and bad edge. On a cycle, the edge with minimum ID among all of the outgoing and bad edges is certain to be eliminated by both of the old fragments connected to it, since they both agree on its elimination. As a result, the cycle will break.

Recall that a 1-bad fragment is either because of cycle or a long path. Let us say the number of 1-bad fragments that have not received ChoiceForbidden on cycle or on top of long path is $k$. Since only edges with smaller ID are removed after the agreement on both sides, the number of removed edges by CycleRemover is at most $k / 2$. Therefore, the asymptotic number of iterations needed for merging fragments still remains the same.

The algorithm FASTST is shown for finding the spanning tree in $O(n)$ rounds and using $O(n \log n \log \log n)$ messages. We do not give the pseudocode for LOGFInDANY. It does the same thing as FINDANY-C except it uses $O(\log n)$ hash functions with the same pipelining technique to get high probability of success in finding outgoing edges.

Over the course of the algorithm, we use $\log ^{*} n$ phases and in each phase we have the threshold $T=n /\left(\log ^{(i)} n\right)^{2}$. In any iteration FINDANY-C and LOGFINDANY spend time proportional to the height of the fragment which is bounded by $T$. Note that using $O(\log n)$ hash functions in a pipelined manner in LOGFINDANY does not affect time complexity. Therefore, similar to the analysis of Algorithm 4, the time complexity is $O(n)$. Moreover, we are using $O(\log n)$ hash functions after the first phase. As in Theorem 5, the overall number of iterations after the phase is $O(\log \log n)$. Hence, the message complexity is $O(n \log n)$ for the first phase, and $O(n \log n \log \log n)$ for the rest of the phases which is $O(n \log n \log \log n)$ overall.

\section{CONCLUSION}

We have shown that time can be brought down to linear in $n$ while maintaining a communication which is sublinear in $m$ when the average degree is $\omega\left(n^{\epsilon}\right)$ for the MST and $\omega(\log n \log \log n)$ for the spanning tree. We have also shown the first minimum spanning tree construction algorithm which uses time approximately proportional to the diameter of the MST without paying an excessive cost for communication.

Some intriguing questions remain: Can time be reduced to $o(n)$ when the network has small diameter, but the diameter of the MST is large, without $m$ communication? Can MST be computed with a deterministic algorithm, or a Las Vegas when nodes do not know the exact size of the network, or in an asynchronous model, with $o(m)$ communication?

\section{REFERENCES}

[Awe87] Baruch Awerbuch. Optimal distributed algorithms for minimum weight spanning tree, counting, leader election, and related problems. In Proceedings of the nineteenth annual ACM symposium on Theory of computing, pages 230-240. ACM, 1987. 

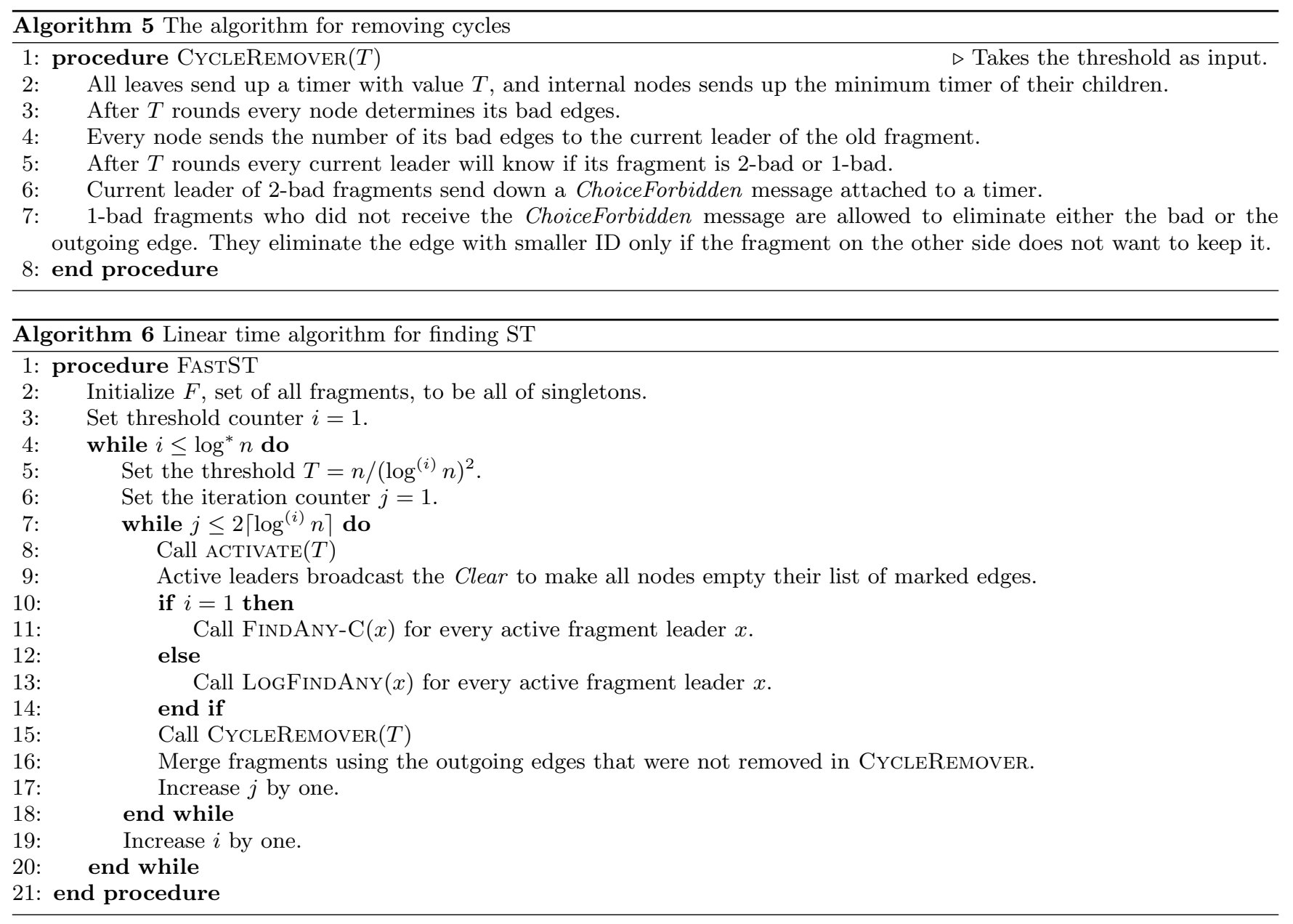

[CT85] F Chin and HF Ting. An almost linear time and o (nlogn+ e) messages distributed algorithm for minimum-weight spanning trees. In Foundations of Computer Science, 1985., 26th Annual Symposium on, pages 257-266. IEEE, 1985.

[Elk04] Michael Elkin. A faster distributed protocol for constructing a minimum spanning tree. In Proceedings of the fifteenth annual ACM-SIAM symposium on Discrete algorithms, pages 359-368. Society for Industrial and Applied Mathematics, 2004.

[Elk06] Michael Elkin. An unconditional lower bound on the time-approximation trade-off for the distributed minimum spanning tree problem. SIAM Journal on Computing, 36(2):433-456, 2006.

[Gaf85] Eli Gafni. Improvements in the time complexity of two message-optimal election algorithms. In Proceedings of the fourth annual ACM symposium on Principles of distributed computing, pages 175-185. ACM, 1985.

[GHS83] Robert G. Gallager, Pierre A. Humblet, and Philip M. Spira. A distributed algorithm for minimum-weight spanning trees. $A C M$ Transactions on Programming Languages and systems (TOPLAS), 5(1):66-77, 1983.
[GKP98] Juan A Garay, Shay Kutten, and David Peleg. A sublinear time distributed algorithm for minimum-weight spanning trees. SIAM Journal on Computing, 27(1):302-316, 1998.

[GP16] Mohsen Ghaffari and Merav Parter. Mst in log-star rounds of congested clique. In Proceedings of the 2016 ACM Symposium on Principles of Distributed Computing, PODC '16, pages 19-28, New York, NY, USA, 2016. ACM.

$\left[\mathrm{HPP}^{+} 15\right]$ James W Hegeman, Gopal Pandurangan, Sriram V Pemmaraju, Vivek B Sardeshmukh, and Michele Scquizzato. Toward optimal bounds in the congested clique: Graph connectivity and mst. In Proceedings of the 2015 ACM Symposium on Principles of Distributed Computing, pages 91-100. ACM, 2015.

[KKT15] Valerie King, Shay Kutten, and Mikkel Thorup. Construction and impromptu repair of an mst in a distributed network with o (m) communication. In Proceedings of the 2015 ACM Symposium on Principles of Distributed Computing, pages 71-80. ACM, 2015.

[KP95] Shay Kutten and David Peleg. Fast distributed construction of k-dominating sets and applications. In Proceedings of the fourteenth 
annual ACM symposium on Principles of distributed computing, pages 238-251. ACM, 1995.

$\left[\mathrm{KPP}^{+} 15\right]$ Shay Kutten, Gopal Pandurangan, David Peleg, Peter Robinson, and Amitabh Trehan. On the complexity of universal leader election. Journal of the ACM (JACM), 62(1):7, 2015.

[LPSPP05] Zvi Lotker, Boaz Patt-Shamir, Elan Pavlov, and David Peleg. Minimum-weight spanning tree construction in o $(\log \log \mathrm{n})$

communication rounds. SIAM Journal on Computing, 35(1):120-131, 2005.

[Pel00] David Peleg. Distributed computing. SIAM Monographs on discrete mathematics and applications, 5, 2000.

[PRS16] Gopal Pandurangan, Peter Robinson, and Michele Scquizzato. A time-and message-optimal distributed algorithm for minimum spanning trees. arXiv preprint arXiv:160\%.06883, 2016.

$\left[\mathrm{SHK}^{+} 12\right]$ Atish Das Sarma, Stephan Holzer, Liah Kor, Amos Korman, Danupon Nanongkai, Gopal Pandurangan, David Peleg, and Roger Wattenhofer. Distributed verification and hardness of distributed approximation. SIAM Journal on Computing, 41(5):1235-1265, 2012. 